\title{
Fault Diagnosis of Helical Gear Box Using Vibration Signals through J-48 Graft Algorithm and Wavelet Features
}

\author{
Nikhil Pawar', V. Sugumaran ${ }^{1}$, Ameet Singh ${ }^{1}$ and M. Amarnath ${ }^{2}$ \\ 'School of Mechanical and building Science (SMBS), VIT University, Chennai Campus, Chennai - 600127, \\ Tamil Nadu, India; nikhil.pawar2012@vit.ac.in, v_sugu@yahoo.com, \\ ameet.singh2015@vit.ac.in \\ 2Department of Mechanical Engineering, Indian Institute of Information Technology Design and Manufacturing, \\ Airport Rd, Jabalpur Campus, Khamaria, Jabalpur - 482005, Madhya Pradesh, India; amarnath.cmy@gmail.com
}

\begin{abstract}
Objectives: In this paper, machine learning approach, grounded on vibrations, has been used for helical gear box and holds a vital position in the industry. This approach has three steps namely feature extraction, feature selection and feature classification. Firstly, feature extraction was carried out using Matrix Laboratory (MATLAB) software. Feature selection was done using J48 classifier. The nodes with highest classification accuracy were further tested using J48 graft classifier and the results obtained were very promising. Methods/Analysis: Vibration signals were obtained from the experimental set up of the helical gear box. The recorded signals were then used for feature extraction using MATLAB through different wavelet features. The total numbers of signals extracted were 448 with each class consisting of 64 signals. The families of wavelets taken into account for fault diagnosis were Haar, Discrete Mayer, Daubechies, Biorthogonal, Reverse Biorthogonal, Coiflet and Symlets (SYM). In wavelet selection, signals were dissected into various frequencies and each was analyzed with appropriate resolution.J48 classifier was used to carry out the feature selection process and decision tree was obtained for Sym 8 wavelet. The best combination of nodes was visualized and further feature classification was done on these nodes. Findings: Feature classification was carried out by J48 graft algorithm. Using the grafting technique, the classifier achieved the highest accuracy for pruned data for 10 times cross validation. It gave maximum accuracy for pruned data (40\%) and the results were satisfactory. Novelty/Improvements: The J48 graft algorithm uses grafting to infer from previous decision trees. This helps in reducing prediction errors.
\end{abstract}

Keywords: Decision Tree, Gearbox Fault Diagnosis, J48 Classifier, J48 graft Classifier, Wavelet

\section{Introduction}

In recent years helical gear box condition monitoring has gained popularity. A significant number of failures occur due to localized defects. Fatigue cracking occurs due to constant cyclic contact stressing, when a major portion of the surface is displaced during operation, leading to localized defects at an initial stage. Helical gear box can operate under a variety of speeds and load conditions', making it is difficult to measure and delineate local defects. Nowadays, fault detection and diagnosis are being carried out by physical parameters such as vibration, acoustic emission and wear debris. Fault classification techniques ${ }^{1}$ have been utilized in a vast array of pattern recognition applications including vibration monitoring, previously used by the author has recorded a model study using numerous physical defects in various environments of velocity and load ${ }^{2}$. Vibration signals ${ }^{\underline{3}}$ collected hold crucial data regarding the working devices. The concept of using vibration ${ }^{4}$ was initially used where vibration signals were used to detect

${ }^{*}$ Author for correspondence 
faults and measure the severity. Many important like biology, ultrasound and thermal imaging ${ }^{5}$ all rely on wavelet analysis ${ }^{6,7}$. "The paper show cases the mother wavelet selection techniques with weight age on the quantitative approaches ${ }^{-}$. Various mother wavelets have been studied in this paper. The various mother wavelets are Haar, Daubechies, coiflet, SYM wavelets for fault detection? Thorough research has shown that the optimal mother wavelet proposed was coiflet wavelet, due to lower sum of coefficients for all values of fault resistances. However, in this study, SYM wavelets were clearly the better option due to higher accuracy. The accuracy of characteristics derived from wavelets for detecting faults of a gear box using Artificial Neural Network (ANN) and Proximal Support Vector Machine (PSVM) ${ }^{10}$. The J48 algorithm was used for classification of the statistical features of Morlet wavelet coefficients. The predominant features were fed as input for training. The relative accuracy in classifying the faults in the bevel gear box was compared using ANN and PSVM. Both ANN and PVSM had a high average classification accuracy of $97.5 \%$ and $97 \%$ respectively. However, PVSM had an edge over ANN due to lesser time required for training. A similar concept is used in this paper, the analysis here was done on a helical gearbox. The paper signifies data classification using Naïve Bayes and J48 classification algorithm ${ }^{11}$. Naive Bayes algorithm is based on probability and $\mathrm{J} 48$ algorithm is based on decision tree $\mathrm{e}^{12}$. A comparative evaluation of the classifiers Naïve Bayes $\underline{13}$ and J48 were done in the paper. The best fit tree is selected from the J48 classifier to carry out further analysis. The effectiveness of the J48 graft classifier is evident from the work presented. Through this paper a comprehensive analysis of various classifiers (both pruned and unpruned) using Weka ${ }^{14,15}$ software was implemented on a spam base dataset. The results were compared based on an evaluation criteria. Naive Bayes algorithm $\frac{16}{16}$ was previously used for carrying out fault diagnosis. The J48 graft classifier, was more complex due to its large tree size and high number of leaves. However, the complexity did not affect its performance as it had a higher classification accuracy. The only drawback presented was the higher time taken by the classifier with respect to J48 and simple cart classifier. This paper uses vibration signals to conduct the fault diagnosis ${ }^{17}$. The performance of the J48 grafts algorithm classification of bank directing marketing ${ }^{18}$. Although its accuracy was not as high as SVM algorithm, the J48 graft classifier still presented a decent output. Among the many tree classifiers namely, least absolute deviation tree, Naïve Bayes tree, random forest tree, best fit tree and simple cart tree, reduced error pruning tree, the J48 graft tree boasted the highest classification accuracy ${ }^{19,20}$ for the fault diagnosis process. The application of the J48 graft classifier can be extended to the fields of medicine and biology. The J48 graft algorithm can be used diagnosis of diabetes disease ${ }^{21}$. The J48 algorithm has been used to generate credit scores and show that the J48graft has the capacity to facilitate migration from existing data systems toward new concise analytic systems and big data ${ }^{22}$.Comparisons between the $\mathrm{J} 48$ graft algorithm ${ }^{23-25}$ with other tree classifiers has been studied."

In the present study, feature extraction ${ }^{26}$ was done using discrete wavelet features. Feature selection ${ }^{27}$ was carried out by $\mathrm{J} 48$ algorithm, the decision tree enables the visualization of the contribution of features for fault diagnosis ${ }^{28}$. Finally feature classification is done with $\mathrm{J} 48$ graft classifier, the results obtained were highly accurate among various tree algorithms used for carrying out fault diagnosis using vibration signals.

\section{Materials and Methods}

"Figure 1 shows the experimental setup. The setup consists of a 5 HP two stage helical gearbox. The gear box is driven by a $5.5 \mathrm{HP}$, 3-phase induction motor with a rated speed of $1440 \mathrm{rpm}$. The inverter drive is responsible for the control of speed of the motor, which is currently operated at $80 \mathrm{rpm}$. The speed of the first stage of the gearbox is $80 \mathrm{rpm}$. With a step-up ratio of $1: 15$, the speed of the pinion shaft in the second stage of the gear box is 1200 $\mathrm{rpm}$. Figure 1(a) summarizes the specifications of the test rig. The pinion is connected to a D.C motor (which is used as generator) to generate $2 \mathrm{~kW}$ power, which is dissipated in a resistor bank. Therefore, the actual load on the gearbox is only $2.6 \mathrm{HP}$ which is $52 \%$ of its rated power $5 \mathrm{HP}$. In industrial environment utilization of load varies from $50 \%$ to $100 \%$. In the case of traditional dynamometer, additional torsional vibrations can occur due to torque fluctuations. This is avoided in this case by using D.C motor and resistor bank. Tyre couplings are fitted between the electrical machines and gear box so that backlash in the system can be restricted to the gears. The motor, gear box and generator are mounted on I-beams, which are anchored to a massive foundation. Vibration signals are measured using a Brüel\&Kjær accelerometer which is installed close to the test bearing. Signals are sampled at a sampling frequency of $8.2 \mathrm{kHz}$. The signals were collected in 8 different classes, each class containing 54 distinct samples. 


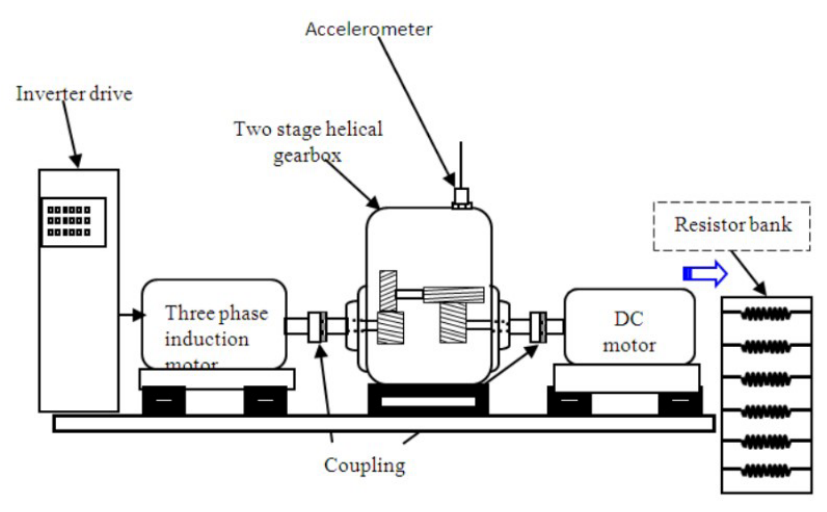

Figure 1. Experimental Setup.

\begin{tabular}{|} 
Table 1: Specifications of Helical gearbox \\
\begin{tabular}{|c|c|c|}
\hline & First stage & Second stage \\
\hline Number of teeth & $44 / 13$ & $73 / 16$ \\
\hline Pitch circle diameter (mm) & $198 / 65$ & $202 / 48$ \\
\hline Pressure angle $\left({ }^{\circ}\right)$ & 20 & 20 \\
\hline Helix angle & 20 & $2.75 / 3$ \\
\hline Modules & $4.5 / 5$ & $1200 \mathrm{rpm}$ (output) \\
\hline Speed of shafts & $80 \mathrm{rpm}$ (input) & $320 \mathrm{~Hz}$ \\
\hline Mesh frequency & $59 \mathrm{~Hz}$ & $1: 15$ \\
\hline Step - up ratio & \multicolumn{2}{|c}{$5 \mathrm{HP}$} \\
\hline Rated power & \multicolumn{2}{|c}{$2.6 \mathrm{HP}$} \\
\hline Power Transmitted & \\
\hline
\end{tabular}
\end{tabular}

Figure 1(a). Specification of helical gear box.

Seeded fault trials are extremely necessary to study the fault detection procedures. Faults can be simulated in the helical gear box using surface grinding, adding iron particles in gearbox lubricant, and using Electric Discharge Machining (EDM) or simply overloading the gear box, which would test it in accelerated conditions. The simplest approach is chip off one edge of the gear. This simulates the partial tooth break ${ }^{29}$. Feature selection was then carried out on the vibration signals using Discrete Wavelet Transform (DWT) algorithm. Out of the numerous wavelets, symlet wavelet was selected due its high classification accuracy. Feature extraction was done by the J48 classifier to evaluate the 8 symlet wavelets. Finally, feature classification carried out on the specific combination of nodes to determine the optimum number of objects for the best classification accuracy ${ }^{30}$. The methodology of the study is given in Figure 2."

\subsection{Feature Extraction}

Fault diagnosis is done through vibration signals using DWT algorithm. Moreover, the signal obtained is timedomain signal. DWT is used to connect the signal belonging to time domain to the time-frequency domain

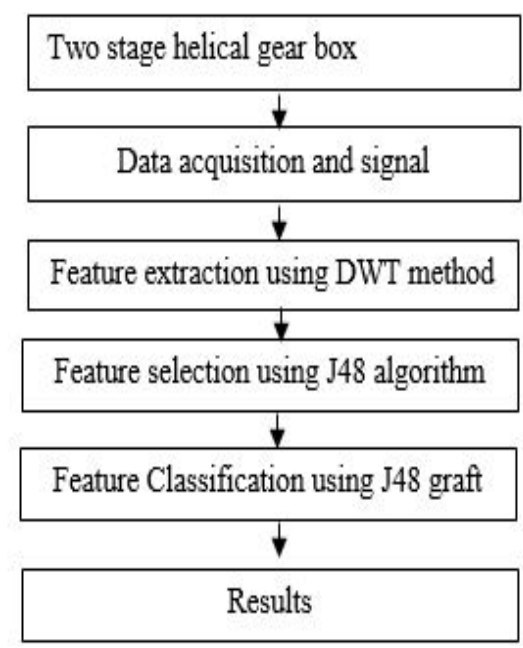

Figure 2. Flowchart of methodology followed.

information. The process of wavelet decomposition was performed on vibration signals using DWT. The trends and details were the consequence of decomposition. For next level trend and detail, the previous trends obtained from decomposition are decomposed again. Additional levels of details are obtained by the decomposition of the preceding trend levels. The length of the signal is 8192 $\left(2^{13}\right)$ and possible decomposition levels are 13. At each level, the detail coefficient was used to compute the energy content using the following formulae:

$\sum_{V_{\mathrm{i}}=i=1}^{n} \mathrm{xi} 2$

Where $x_{\mathrm{i}=}$ details coefficients $n=$ number of detail coefficients.

Then the features were defined as the energy content at each level. The feature vector is defined as $V=(v 1, v 2, v 3, . . v m)$

When $m-($ number such that length of signal $)=2^{\mathrm{m}}$

$v 1, v 2, v 3 \ldots$ are energy content at given level

Families of wavelets taken into account for the fault diagnosis are:

1. Haar wavelet

2. Discrete Meyer wavelet

3. Daubechies wavelet - Db1,db2, db3, db4, db5, db6, $\mathrm{db} 7, \mathrm{db} 8, \mathrm{db} 9, \mathrm{db} 10$

4. Biorthogonal wavelet - bior 1.1, bior 1.3, bior 1.5, bior 2.2 , bior 2.4, bior 2.6, bior 2.8, bior 3.1, bior 3.3, bior 3.5, bior 3.7, bior 3.9, bior 4.4, bior 5.5, bior 6.8

5. Reversed Biorthogonal wavelet - rbio1.1, rbio 1.3, rbio 1.5, rbio 2.2, rbio 2.4, rbio 2.6, rbio 2.8, rbio 3.1, rbio 3.3, rbio 3.5, rbio 3.7, rbio 3.9, rbio 4.4, rbio 5.5, rbio 6.8 
6. Coiflet - coif 1 , coif 2 , coif 3 , coif 4 , coif 5

7. Symlets - sym $2, \operatorname{sym} 3$, sym 4 , sym 5 , sym $6, \operatorname{sym} 7$, sym 8

\subsection{Wavelet Selection}

Fifty four distinct wavelets were selected by using mother wavelet selection technique, from sym 2 ,sym 3 ,sym 4 , sym 5,sym 6, sym 7 and sym 8 were studied and a comparative analysis was carried out using J48 algorithm.Sym8 was selected based on the highest accuracy obtained as shown is Figure 8."

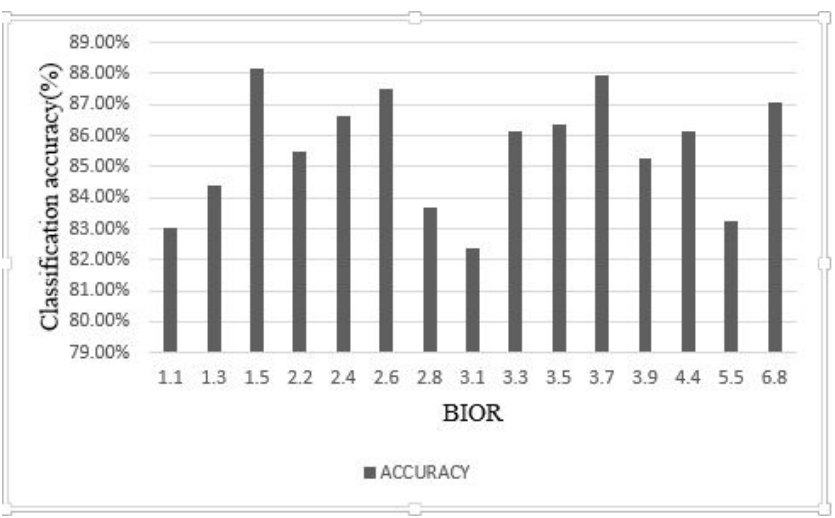

Figure 3. BIOR wavelet versus classification accuracy.

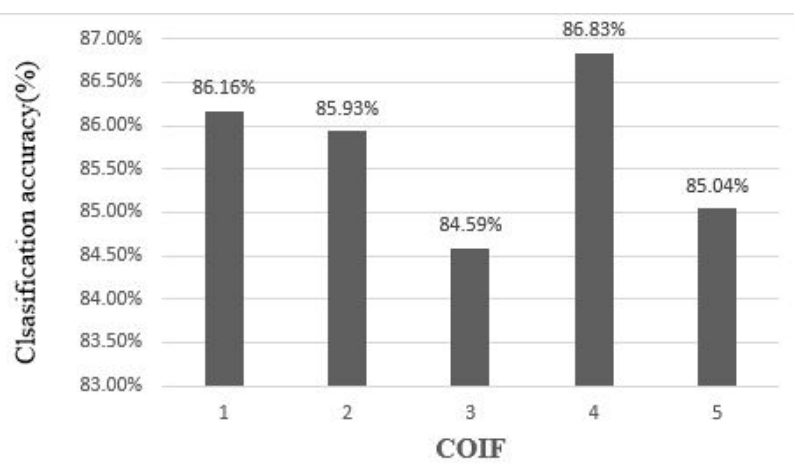

Figure 4. COIFLET wavelet versus classification accuracy.

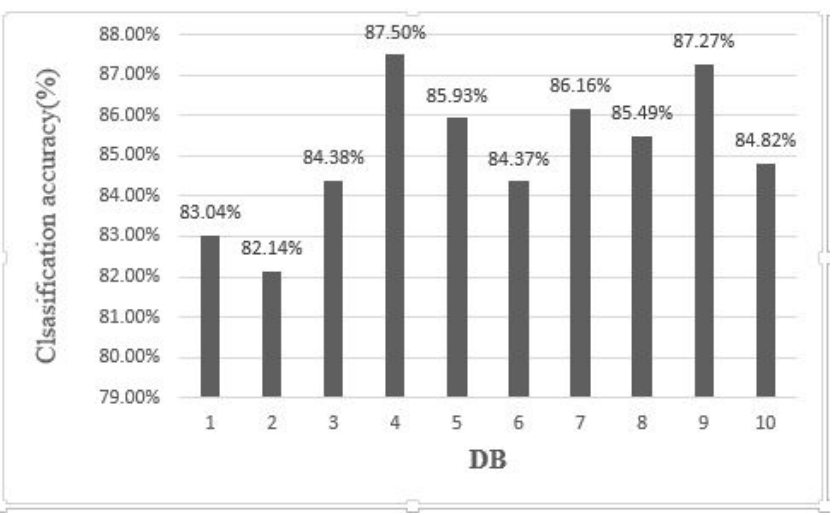

Figure 5. DB wavelet versus classification accuracy.

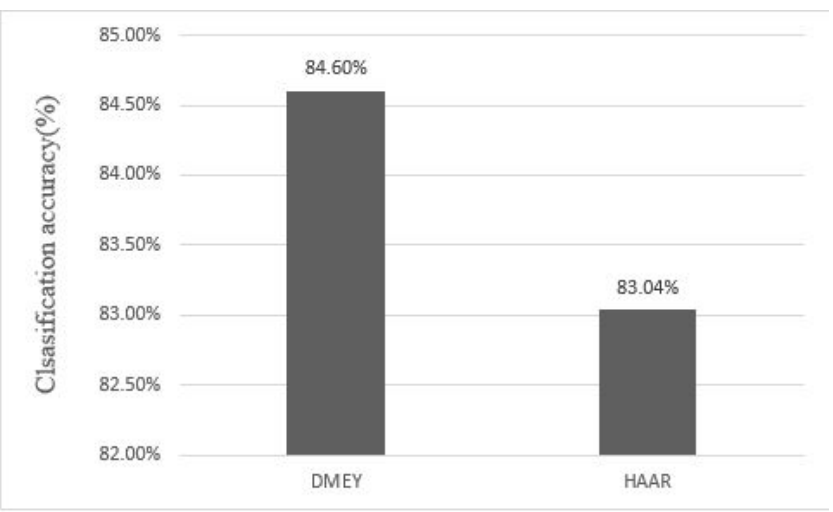

Figure 6. DMEY and HAAR versus classification accuracy.

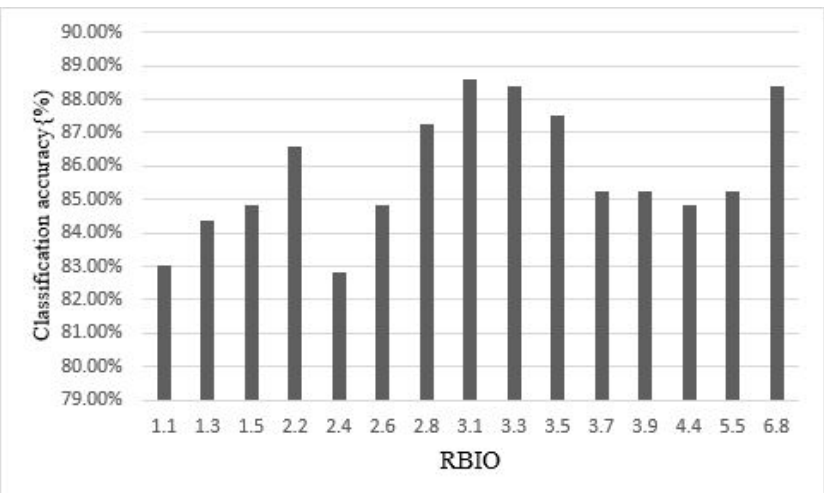

Figure 7. RBIO wavelet versus classification accuracy.

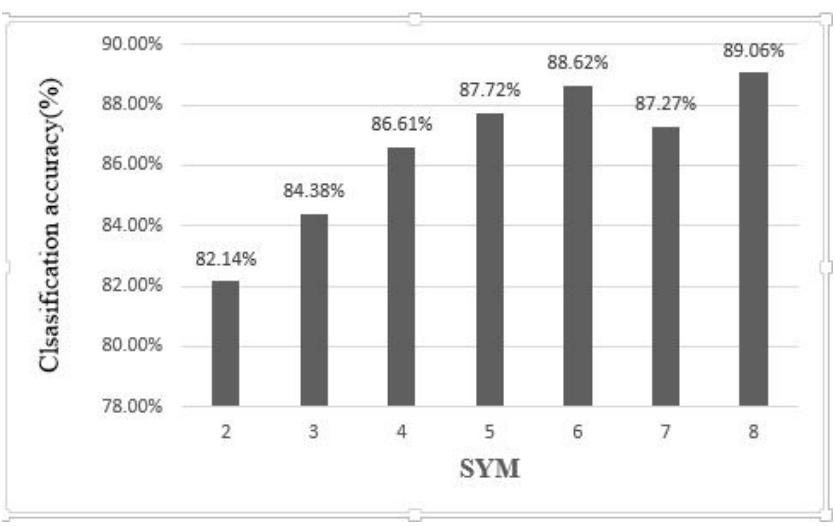

Figure 8. SYM wavelet versus classification accuracy.

\subsection{Feature Selection}

A Decision Tree is used to understand the relationship between a dependent attribute (variable) given the values of the independent (input) attributes (variables). This concept aids modeling and knowledge extraction from the bulk data available $e^{31}$. J48 algorithm was used to carry out the feature selection process. It creates a binary tree which is useful in the classification problem. Once 
the tree is built, it is applied to each ordered list in the database and results in classification for that list. SYM 8 wavelet data was used and classification was carried out by using J48 tree algorithm. Hence, decision tree was obtained through visualization of decision tree.

$\mathrm{V} 3$ is the top node, followed by the combination of nod $\mathrm{es,}, \mathrm{v}+\mathrm{v} 10, \mathrm{v} 3+\mathrm{v} 10+\mathrm{v} 1, \mathrm{v} 3+\mathrm{v} 10+\mathrm{v} 1+\mathrm{v} 6, \mathrm{v} 3+\mathrm{v} 10+\mathrm{v} 1+\mathrm{v} 6+\mathrm{v} 5, \mathrm{v} 3$ $+\mathrm{v} 10+\mathrm{v} 1+\mathrm{v} 6+\mathrm{v} 5+\mathrm{v} 4, \mathrm{v} 3+\mathrm{v} 10+\mathrm{v} 1+\mathrm{v} 6+\mathrm{v} 5+\mathrm{v} 4+\mathrm{v} 2, \mathrm{v} 3+\mathrm{v} 10+\mathrm{v} 1$ $+\mathrm{v} 6+\mathrm{v} 5+\mathrm{v} 4+\mathrm{v} 2+\mathrm{v} 7, \mathrm{v} 3+\mathrm{v} 10+\mathrm{v} 1+\mathrm{v} 6+\mathrm{v} 5+\mathrm{v} 4+\mathrm{v} 2+\mathrm{v} 7+\mathrm{v} 8, \mathrm{v} 3+\mathrm{v}$ $10+\mathrm{v} 1+\mathrm{v} 6+\mathrm{v} 5+\mathrm{v} 4+\mathrm{v} 2+\mathrm{v} 7+\mathrm{v} 8+\mathrm{v} 9, \mathrm{v} 3+\mathrm{v} 10+\mathrm{v} 1+\mathrm{v} 6+\mathrm{v} 5+\mathrm{v} 4+\mathrm{v} 2$ $+\mathrm{v} 7+\mathrm{v} 8+\mathrm{v} 9+\mathrm{v} 11, \mathrm{v} 3+\mathrm{v} 10+\mathrm{v} 1+\mathrm{v} 6+\mathrm{v} 5+\mathrm{v} 4+\mathrm{v} 2+\mathrm{v} 7+\mathrm{v} 8+\mathrm{v} 9+\mathrm{v} 11$ $+\mathrm{v} 12, \mathrm{v} 3+\mathrm{v} 10+\mathrm{v} 1+\mathrm{v} 6+\mathrm{v} 5+\mathrm{v} 4+\mathrm{v} 2+\mathrm{v} 7+\mathrm{v} 8+\mathrm{v} 9+\mathrm{v} 11+\mathrm{v} 12+\mathrm{v} 13$.

Feature selection was done for all 13 features. Further tests took place using the $\mathbf{J} 48$ algorithm to identify the sequence of nodes and the node with the best accuracy. The nodes, $\mathrm{v} 3+\mathrm{v} 10+\mathrm{v} 1+\mathrm{v} 6+\mathrm{v} 5+\mathrm{v} 4+\mathrm{v} 2$ had an accuracy of 89.2857 i.e. the maximum, among the 13 combinations. The Figure 10 shown compares the accuracies obtained by the different combination of nodes and helps identify the node with the highest accuracy."

\subsection{Feature Classification}

A grafted decision tree is generated by the J48 graft algorithm from a J48 tree algorithm. The grafting technique

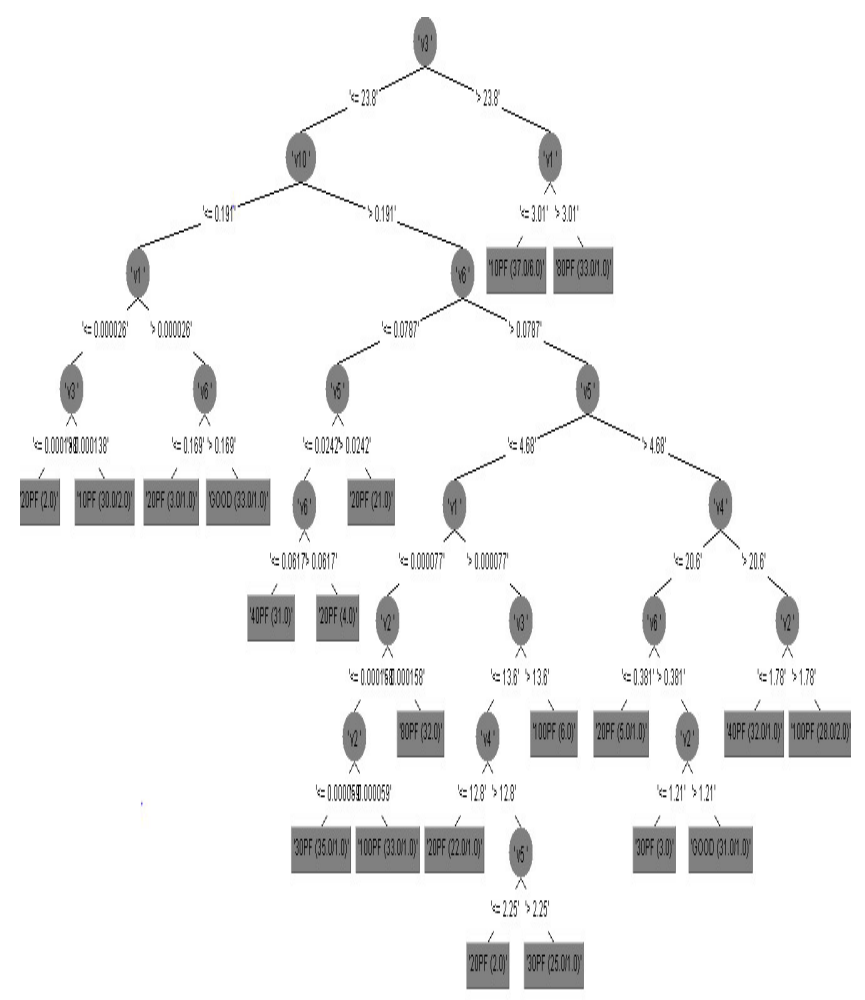

Figure 9. J48 decision tree. is a post process technique that adds nodes to inferred decision trees with the purpose of reducing prediction errors. This process only allows branching that avoids the introduction of classification errors into data that have previously been correctly classified. As a result, rather than introducing errors, the grafting technique eliminates. For evaluation purposes the following terms have been used: True positive (TP) for correctly identified, True Negative (TN) for correctly rejected, and False Positive (FP) for incorrectly identified, Precision, Recall, F-Measure, and Accuracy. Recall is referred to as the True Positive Rate or Sensitivity, and precision is Positive Predictive Value (PPV); True Negative Rate is Specificity. F-measure is a measure of a test's accuracy. F-measure reaches its best value at 1 and worst score at 0 . The $\mathrm{v} 3+\mathrm{v} 10+\mathrm{v} 1+\mathrm{v} 6+\mathrm{v} 5+\mathrm{v} 4+\mathrm{v} 2$ nodes were tested using the J48 Graft classifier, by varying the minimum number of objects to pinpoint the optimum number of objects with the highest accuracy.

The graph depicts a uniform trend of the accuracy on increasing the number of objects. The highest accuracy of $90.17 \%$ is obtained initially with the number of objects equal to 1 . This is succeeded by a steady decline for the next

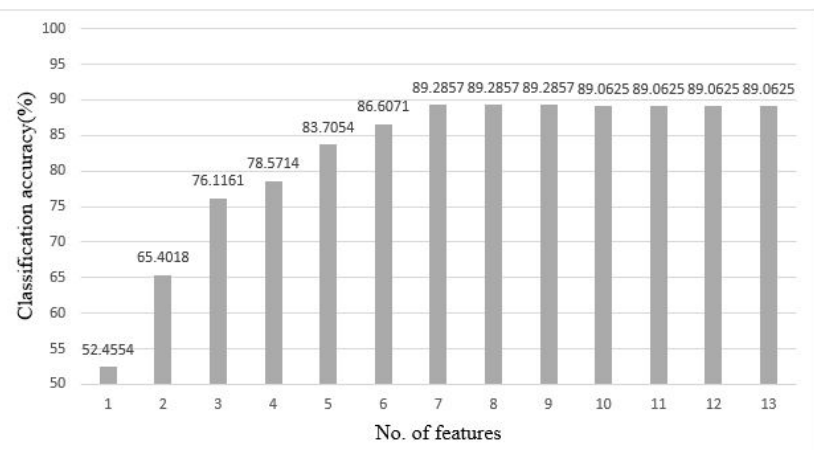

Figure 10. Accuracy versus SYM nodes.

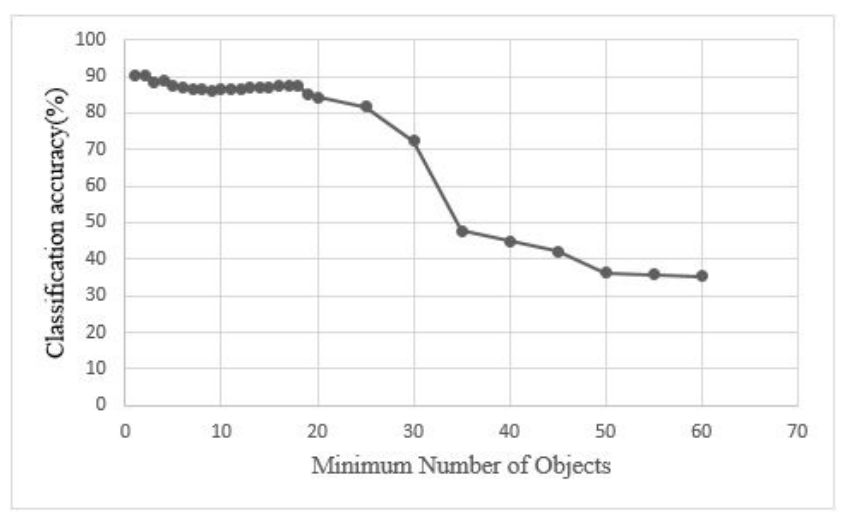

Figure 11. Accuracy versus minimum number of objects. 
ten objects were accuracy values equal or as low as $86.60 \%$. There is a minor increase in accuracy after the $12^{\text {th }}$ object with highest accuracy of $87.50 \%$ during this period. Finally, there is a steep decline after the $20^{\text {th }}$ object where the accuracy decreases non uniformly and rapidly."

\section{Results and Discussion}

Vibration signals from a helical gearbox were recorded. 54 discrete wavelets were obtained and were used to carry out feature extraction. The signals were divided into 8 distinct groups (symlets), each symlet containing 54 different wavelet samples. These were tested in the Weka software using the J48 classifier algorithm. The group with the highest accuracy was visualized using the decision tree. The nodes of the tree were separately evaluated to learn about their individual accuracies. Upon, deriving the nodes with the maximum accuracy, the J48 Graft algorithm helped to identify the optimum number of objects for the given nodes.

\subsection{Feature Classification}

The vibration signals were recorded for normal and abnormal conditions of helical gear box. Totally 448 samples were collected; out of which 64 samples were from healthy condition. For faulty gears with $10 \%, 20 \%, 40 \%$, $60 \%, 80 \%$ and $100 \%$ fault, 64 samples from each condition were collected. The wavelet features were treated as features (attributes) were inputs to the algorithm. Numerous conditions ( $10 \%$ fault, $20 \%$ fault, $40 \%$ fault, $60 \%$ fault, $80 \%$ fault, $100 \%$ fault and healthy) of the classified data were the outputs. Although the nodes closer to the root are more significant, all nodes in the tree are given equal importance for feature subset selection in order to maintain simplicity of the code $\mathrm{j}^{32,33}$.

The interpretation of the confusion matrix is as follows (Figure 12). The diagonal elements in the confusion matrix above, show the number of correctly classified instances.

1. In the first row, the first element shows number of data points that belong to 'Good' class and classified by the classifier as 'Good'.

2. In the first row, the fourth element shows the number of data points belonging to 'Good' class but misclassified as ' $30 \%$ fault'.

3. In the first row, the seventh element shows the number of 'Good' data points misclassified as ' $100.0 \%$ fault'.
4. In the first row, fifth and sixth elements represent misclassification of faulty conditions, denoted by ' 0 '.

5. However, there are misclassifications in other conditions. They are given in non-diagonal elements. Here, out of 448 data points, 44 data points were misclassified by the algorithm.

The summary of stratified cross validation obtained from the confusion matrix is given below-

Total number of instances

Correctly classified instances

$40490.17 \%$

Incorrectly classified instances 44

$9.82 \%$

The detailed class-wise accuracy of the J48 graft algorithm is presented in Figure 3. The TP rate stands for true positive and its value should be close to 1 and FP rate stands for false positive and its value should be close to 0 for better classification accuracy, which is confirmed in the paper ${ }^{34}$.

\begin{tabular}{|l|l|l|l|l|l|l|l|}
\hline Good & $10 \mathrm{PF}$ & $20 \mathrm{PF}$ & $30 \mathrm{PF}$ & $40 \mathrm{PF}$ & $80 \mathrm{PF}$ & $100 \mathrm{PF}$ & Classified \\
\hline 60 & 0 & 2 & 1 & 0 & 0 & 1 & as \\
\hline 1 & 56 & 2 & 2 & 0 & 2 & 1 & $10 \mathrm{PF}$ \\
\hline 0 & 10 & 50 & 2 & 0 & 0 & 2 & $20 \mathrm{PF}$ \\
\hline 2 & 0 & 2 & 56 & 3 & 0 & 1 & $30 \mathrm{PF}$ \\
\hline 1 & 0 & 1 & 2 & 57 & 0 & 3 & $40 \mathrm{PF}$ \\
\hline 0 & 0 & 1 & 0 & 0 & 63 & 0 & $80 \mathrm{PF}$ \\
\hline 0 & 1 & 0 & 1 & 0 & 0 & 62 & $100 \mathrm{PF}$ \\
\hline
\end{tabular}

Figure 12. Confusion matrix of best fit tree.

\begin{tabular}{|c|c|c|c|c|c|c|c|}
\hline & TP & FP & Precision & Recall & F- & ROC- & Class \\
& Rate & Rate & & & Measure & Area & \\
\hline & 0.938 & 0.01 & 0.938 & 0.938 & 0.938 & 0.972 & GOOD \\
\hline & 0.875 & 0.029 & 0.836 & 0.875 & 0.855 & 0.938 & $10 \mathrm{PF}$ \\
\hline & 0.781 & 0.021 & 0.862 & 0.781 & 0.82 & 0.957 & $20 \mathrm{PF}$ \\
\hline & 0.875 & 0.021 & 0.875 & 0.875 & 0.875 & 0.939 & $30 \mathrm{PF}$ \\
\hline & 0.891 & 0.008 & 0.95 & 0.891 & 0.919 & 0.942 & $40 \mathrm{PF}$ \\
\hline & 0.984 & 0.005 & 0.969 & 0.984 & 0.977 & 0.99 & $80 \mathrm{PF}$ \\
\hline & 0.969 & 0.021 & 0.886 & 0.969 & 0.925 & 0.972 & $100 \mathrm{PF}$ \\
\hline Weighted & 0.902 & 0.016 & 0.902 & 0.902 & 0.901 & 0.958 & \\
\hline
\end{tabular}

Figure 13. Detailed accuracy table of best fit tree. 


\section{Conclusion}

Gears are important machine elements which undergoes constant wear. This paper presents an algorithm based interpretation of vibration signals for fault diagnosis of helical gear box. DWT was used with different wavelets. Sym 8 wavelet was selected among these wavelets due to its high accuracy. J48 decision tree classifier was used to carry out the feature selection process. The decision tree was studied and the sequences of nodes were visualized. Feature classification was carried out by J48 graft algorithm. Using the grafting technique, the classifier achieved the highest accuracy for pruned data for 10 times cross validation. It gave maximum accuracy for pruned data (40\%) and the results were satisfactory."

\section{References}

1. Pravin KA, Jegadeeshwaran R, Sugumaran V. Roller bearing fault diagnosis by decision tree algorithms with statistical feature. International Journal of Research in Mechanical Engineering. 2013 Jul-Sep; 1(1):1-9.

2. Chokkalingam SP, Komathy K. Comparison of different classifier in WEKA for rheumatoid arthritis. In the International Conference on Human Computer Interactions (ICHCI). Institute of Electrical and Electronics Engineers (IEEE); 2013 Aug 23-24. p. 1-6.

3. Rajput S, Arora A. Designing spam model- classification analysis using decision trees. International Journal of Computer Applications. 2013; 75(10):2.

4. Wisaeng K. A comparison of different classification techniques for bank direct marketing. International Journal of Soft Computing and Engineering. 2013 Sep; 3(4):116-9.

5. Goyal A, Mehta R. Performance comparison of naïve bayes and $\mathbf{J} 48$ classification algorithms. International Journal of Applied Engineering Research. 2012; 7(11):1-5.

6. Kokare A, Venkatesan P, Tandel S, Palivela H. Survey on classification based techniques on non-spatial data. International Journal of Innovative Research in Science, Engineering and Technology. 2014 Feb; 3(1):409-13.

7. Hayashi Y, Tanaka Y, Takagi T, Saito T, Iiduka H, Kikuchi H, Bologna G, Mitra S. Recursive rule extraction algorithm with J48 graft and applications to generating credit scores. Journal of Artificial Intelligence and Soft Computing Research. 2016; 6(1):35-44.

8. Choubey DK, Paul S. GA_J48graft DT: a hybrid intelligent system for diabetes disease diagnosis. International Journal of Bio-Science and Bio-Technology. 2015; 7(5):135-50.

9. Arora V, Siddiqui JA, Mulaveesala R, Muniyappa A. Hilbert transform-based pulse compression approach to infrared thermal wave imaging for sub-surface defect detection in steel material. Insight-Non-Destructive Testing and Condition Monitoring. 2014 Oct; 56(10):550-2.

10. Katore LS, Umale JS. Comparative study of recommendation algorithms and systems using WEKA. International Journal of Computer Applications. 2015 Jan; 110(3):14-7.

11. Yesilyurt. Gearbox fault detection and severity assessment using vibration analysis [PhD Thesis]. UK, University of Manchester; 1997.

12. Yadav RB, Jha B, Rao KRM, Yadav HL. Selection of optimal mother wavelet for fault detection using discrete wavelet transform. International Journal of Advanced Research in Electrical, Electronics and Instrumentation Engineering. 2013 Jun; 2(6):2338-43.

13. Tse PW, Yang W, Tam HY. Machine fault diagnosis through an effective exact wavelet analysis. Journal of Sound and Vibration. 2004; 277:1005-24.

14. Santra AK, Christy CJ. Genetic algorithm and confusion matrix for document clustering. International Journal of Computer Science Issues. 2012 Jan; 9(1):322-8.

15. Serasiya SD, Chaudhary N. Simulation of various classifications results using WEKA. International Journal of Recent Technology and Engineering. 2012 Aug 5; 1(3):155-62.

16. Tripathi G, Naganna S. Feature selection and classification approach for sentiment analysis. Machine Learning and Applications: An International Journal(MLAIJ). 2015 Jun; 2(2):1-16.

17. Vijayarani S, Muthulakshmi M. Comparative analysis of bayes and lazy classification algorithms. International Journal of Advanced Research in Computer and Communication Engineering. 2013 Aug; 2(8):3118-24.

18. Patil TR, Sherekar SS. Performance analysis of naive bayes and J48 classification algorithm for data classification. International Journal of Computer Science and Applications. 2013 Apr; 6(2):256-61.

19. Khatun A, Chowdhury MMH. Image compression using discrete wavelet transform. International Journal of Computer Science Issues. 2012 Jul; 9(4):327-30.

20. Sharma A, Amarnath M, Kankar PK. Feature extraction and fault severity classification in ball bearings. Journal of Vibration and Control. 2016; 22(1):176-92.

21. Kumar H, Kumar TAR, Amarnath M, Sugumaran V. Fault diagnosis of bearings through vibration signal using bayes classifiers. International Journal of Computer Aided Engineering and Technology. 2014 Jan; 6(1):14-28.

22. Amarnath, Jain, Sugumaran, Kumar H. Fault diagnosis of helical gear box using naïve bayes and bayes net. International Journal of Decision Support Systems. 2015; $1(1): 4-17$.

23. Sharma G, Bhargava N, Bhargava R, Mathuria M. Decision tree analysis on $\mathrm{J} 48$ algorithm for data mining. International Journal of Advanced Research in Computer Science and Software Engineering. 2013 Jun; 3(6):1114-9. 
24. Gangadhar N, Kumar H, Narendranath S, Sugumaran V. Fault diagnosis of single point cutting tool through vibration signal using decision tree algorithm. International Conference on Advances in Manufacturing and Materials Engineering, Procedia Materials Science. 2014; 5:1434-41.

25. Joshuva A, Sugumaran V, Amarnath M. Selecting kernel function of support vector machine for fault diagnosis of roller bearings using sound signals through histogram features. International Journal of Applied Engineering Research. 2015; 10(68):482-7.

26. Byrtus M, Zeman V. On modeling and vibration of gear drives influenced by nonlinear couplings, Mechanism and Machine Theory. 2011 Mar; 46(3):375-97.

27. Saravanan N, Siddabattuni VNSK, Ramachandran KI. Fault diagnosis of spur bevel gear box using Artificial Neural Network (ANN) and Proximal Support Vector Machine (PSVM), Applied Soft Computing. 2010 Jan; 10(1):344-60.

28. Joshuva A, Sugumaran V. Fault diagnostic methods for wind turbine: a review. Asian Research Publishing Network (ARPN) Journal of Engineering and Applied Sciences. 2016 Apr; 11(7):4654-68.

29. Muralidharan V, Sugumaran V, Sakthivel NR. Wavelet decomposition and support vector machine for fault diagnosis of monoblock centrifugal pump. International Journal of Data Analysis Techniques and Strategies. 2011; 3(2):159-77.

30. Ngui WK, Leong MS, Hee LM, Abdelrehman AM. Wavelet analysis: Mother wavelet selection methods. Applied Mechanics and Materials. 2013; 393:953-8.

31. Amarnath M, Jain D, Sugumaran V, Kumar H. Fault diagnosis of helical gear box using decision tree and best-first tree. International Journal of Research in Mechanical Engineering. 2013 Jul-Sep, 1(1):22-33.

32. Villa LF, Renones A, Peran JR, Miguel LJ. Statistical fault diagnosis based on vibration analysis for gear test-bench under non-stationary conditions of speed and load. Mechanical Systems and Signal Processing. 2012 May; 29:436-46.

33. Muralidharan A, Sugumaran V, Soman KP, Amarnath M. Fault diagnosis of helical gear box using variational mode decomposition and random forest algorithm. SDHM: Structural Durability and Health Monitoring. 2014; 10(1):81-97.

34. Arora R, Suman. Comparative analysis of classification algorithms on different datasets using WEKA. International Journal of Computer Applications. 2012 Sep; 54(13):21-5. 